\title{
The Framework of Combining Artificial Intelligence and Construction 3D Printing in Civil Engineering
}

\author{
Kang Tan \\ Dalian University of Technology, College of Civil Engineering, No.2 Linggong Road, Dalian City, P.R.China
}

\begin{abstract}
Construction 3D printing is becoming a significant force driving the transformation of civil engineering industry, but facing many problems for its immaturity. At the same time, the development of artificial intelligence and other advanced technology, especially BIM and virtual reality technology, provides new methods for solving these problems. Based on the defects of construction 3D printing and the advantages of these technology, this paper proposes a framework that combines artificial intelligence with construction 3D printing in five aspects: materials for 3D printing, automation design for 3D printing, digital construction for 3D printing, robots for 3D printing and BIM platform system for 3D printing. BIM platform system associates with the other aspects to work together efficiently during the life cycle of construction.
\end{abstract}

\section{Introduction}

In recent years, construction $3 \mathrm{D}$ printing is one of effective methods to build in a digital way, which brings great changes to the civil engineering industry. Construction 3D printing adopting the principle of layered materials, based on building information model(BIM), decomposes the model into layers with a certain thickness in one direction, then generates the corresponding algorithm and finally completes the automatic construction by the numerical control system in a specified path. Obviously, construction 3D printing is a multidisciplinary area, containing a variety of technologies such as numerical control, material molding and construction management.

Artificial intelligence is a technology that develops theories, methods, techniques, and applications to extend people's intelligence. Artificial intelligence is quite extensive that consists of various parts such as machine learning and computer vision. In general, the goal of it is to make the machine be capable of doing complex work which requires human intelligence in the past.

BIM is not only a kind of technical tools, but also a kind of management tools. It is designed to integrate all the concerned parts into a system in order to manage efficiently. For many projects, artificial intelligence combined with BIM will provide useful reference for decision making. Nowadays, BIM is mainly applied in design and construction, but we do not obtain information about structural damage or safety evaluation. For this reason, the integration between BIM and artificial intelligence is significantly important. It not only provides a visual platform for construction, but also provides vital reference for the management during life cycle of the infrastructure.

With the continuous development of artificial intelligence and BIM, at the same time, construction 3D printing, data mining, virtual reality(VR) and augmented reality(AR) are also flourishing. How to integrate all these advanced technology into a system in order to drive the transformation of traditional construction and improve efficiency of operation has become a hot research topic. Currently, construction 3D printing is immature, there are still many problems in theory, methods and applications. Artificial intelligence provides a new solution for this problem by applying a number of technologies, such as machine learning and computer vision, to construction $3 \mathrm{D}$ printing. This article proposes the framework in five aspects: materials for $3 \mathrm{D}$ printing, automation design for 3D printing, digital construction for $3 \mathrm{D}$ printing, robots for $3 \mathrm{D}$ printing and BIM platform system for 3D printing.

\section{The former research of construction 3D printing and artificial intelligence}

Construction 3D printing, proposed by Joseph Pegna, was a method for the construction of free-form components through cementitious material layer accumulation and selective solidification. The method derived from emerging field of additive manufacturing processes is investigated for its potential effectiveness in construction automation [1]. Behrokh Khoshnevis, a professor at the University of Southern California, presenting 'Contour Crafting' that enabled the layered printing of concrete by large three-dimensional extrusion devices. Contour 
crafting $(\mathrm{CC})$ is a layered fabrication technology that has a great potential in automated construction of whole structures as well as subcomponents [2]. Monolite, a company in UK, put forward a new construction 3D printing method called 'D-shape' that selectively hardened and accumulated gravel powder layer by layer. This method divided structure model with horizontal parallel planes in predetermined path, prearranging an apparatus that deposits in alternation a layer of granular material and a liquid binder only in coincidence with the solid portions [3]. Richard Buswell at Loughborough University proposed 'Concrete Printing', which had higher three-dimensional freedom and smaller stacking resolution. In addition, he explores the issues surrounding design, data and process in terms of the impact of rapid manufacturing on conventional manufacturing [4]. Rouhana studied the effectiveness of this technique in reducing construction duration using computer simulation and comparing the conventional construction method and Contour Crafting. The findings suggested that contour crafting can reduce project duration due to automate the construction process [5]. Mechtcherine adapted a concrete pump to deliver material to specific positions autonomously and attached to the boom accurately with the help of custom-developed printing head [6]. Comparing the value stream maps revealed a $60 \%$ reduction of production time. Further analysis unveiled reduction in construction cost, especially labour cost, minimization of different types of waste as well as improvement of quality [7].

Artificial intelligence is applied to 3D printing in an intelligent, efficient, high quality and service-oriented production process and the printability of 3D objects can be determined by using machine learning. The prefabrication is accelerated through parallel slicing algorithms and the path planning is optimized intelligently. In the aspect of service and security, intelligent demand matching and resource allocation algorithms enable a cloud service platform and evaluation model to provide clients with an on-demand service and access to the collection of shared resources [8]. Yang presented a rapid design method of product modeling and structure condition selecting based on interactive genetic algorithm and 3D printing technology, which gave a design pattern to meet the function needs [9]. Makagonov proposed a solution to the problem of detecting collisions in the additive process of $3 \mathrm{D}$ printing through two methods, which are used in computer vision for recognition: it is the machine learning and tracking based on singular points [10]. Wu detected malicious infill defects in $3 \mathrm{D}$ printing process and the data extracted from images was input to two machine learning algorithms, Naive Bayes Classifier and J48 Decision Trees [11].

\section{Materials for 3D printing}

\subsection{Performance requirements of materials}

Compared with traditional properties of building materials, 3D printing materials should have controllable setting time, appropriate workability and superior mechanical properties. Application of P.O and R-SAC mortar for 3D printing in construction was investigated by Lin [12]. Different key powder parameters such as particle size distribution, powder bed surface quality, powder true/bulk densities, powder bed porosity, and binder droplet penetration behavior were used to quantitatively evaluate the printability of prepared geopolymer-based material [13]. Lediga presented an investigation into the criteria for an optimum concrete mix design to be used in a 3D concrete printing machine [14]. Shakor demonstrates the properties of different concrete mixture designs, showing their performance when used filament in 3D printing and representing a comparison of the results [15].

According to the past research, concrete and gravel powder are two materials used in construction 3D printing, they will be built on principle of layered stacking. The former is self-solidification accumulation, the latter is bonded with gravel powder. The defect of these materials is that their tensile properties are poor. Once tensile stress exceeds the tensile strength, they tend to crack, which may lead to serious damage to structure. Therefore, materials for 3D printing should have good anti-cracking ability, which can restrict the cracks caused by load. As the printing materials need to be transported through the pipe, they should be of good workability. During the process of printing, the upper layer of materials accumulation will impose pressure on the lower layer. If the lower layer does not form enough strength in a short time, it will deform under the pressure. To conclude, the printing materials should have faster initial setting time and higher initial setting strength.

\subsection{Application of artificial intelligence to design 3D printing materials}

The use of artificial intelligence to design new materials may lead to the revolution of materials, which is also suitable for 3D printing materials. To meet above requirements, new materials should be developed with these features: high compression and tensile properties, strong crack resistance and toughness, faster initial setting time and higher initial setting strength. Specifically, artificial intelligence is used to design new materials in following steps. Firstly, a large database is set up to record all the current materials information, including materials components, preparation methods and performance characteristics. Secondly, machine learning algorithms are used to select the materials with ideal performance according to the given requirements. Then the materials in database are compared and analyzed, figuring out the best materials after pattern recognition and extraction. The advantage of machine learning algorithms is that the failed experimental data can also be used as input for next, and then the algorithms are refined.

\section{Automation design for 3D printing}

In the early stage of project, artificial intelligence deals with a large number of relevant case data so as to determine whether the current project has good economic 
benefits. At the same time, the combination of artificial intelligence and BIM can also help owners to set project target costs through materials classification in order to provide precise budget for target cost control. In this way, materials can be finely managed, the quantity of materials required according to the schedule can be rationally guaranteed, and the amount of material used in each area can be reasonably controlled to reduce the waste caused by second handling.

Artificial intelligence can help architects complete site analysis, planning and architectural design, greatly reducing the workload of architectural design. It not only can be applied to early stages of design, including geographical location, climate, geometry, spatial relationships and conceptual design, but also further design and construction plan. After training a large number of data through related machine learning algorithms, artificial intelligence have ability to design. As long as people accurately describe their expectations, artificial intelligence can generate a variety of options for them to choose. In addition, with the help of rapid data analysis and computer vision, it will be much more efficient than humans.

The current method of structural design is mainly based on traditional buildings, but 3D printing building is a new type of structure. Therefore, many new problems should be taken into consideration in its design. In this case, artificial intelligence can learn the principle and method of structural design through a certain algorithm, and then complete the parametric modeling. After analyzing and comparing a variety of design schemes automatically, reasonable scheme will be selected. For example, taking the design of beam as an example, a number of different schemes can be generated to meet the given requirements such as strength, stiffness and stability.

The mechanical and electrical equipment should not only be designed for long-term operation, but as green as possible. It will be achieved by using artificial intelligence algorithms to operate the equipment both efficiently after learning a lot of data. In this way it can not only realize low energy consumption and high efficiency, but also intelligent controlling and recovery. The realization of these functions requires the application of artificial intelligence algorithms for their ability to control complex events and adjust system parameters.

\section{Digital construction for 3D printing}

During the construction phase, first of all, artificial intelligence assists people in making schedule plan, integrating data with BIM model, and dynamically displaying schedule plan in BIM model, so as to realize collaborative management. Secondly, BIM can simulate and optimize the construction plan. With features of visualization, each step in the construction process is simulated. For example, the arrangement can simulate the construction process of masonry and obtain the least amount and maximum efficiency. Third, BIM guides workers during construction. It can reduce the time delay and improve the quality of the project by using the BIM with various professional applications. For example, when installing pipelines, workers can use manual roaming or path roaming to acquire properties of components or local areas, so that collision of the actual construction can be found in advance, and the construction progress can be accelerated.

Digital construction for 3D printing is the most challenging and difficult process, involving wide range of aspects and complex conditions. The application of artificial intelligence to the construction process accomplishes the digital construction by adjusting to actual situation. First, artificial intelligence learns a large number of actual construction cases, and then revise the corresponding construction scheme based on the characteristics of the $3 \mathrm{D}$ printing. In addition, the robot should be able to automatically identify the characteristics of the environment by using advanced algorithm, then select the best plan in short time. After the completion of construction, the robot should be able to detect the quality of the construction and provide timely feedback.

Specifically, artificial intelligence is mainly used in the material identification and the optimization in construction. Firstly, it will judge the quality and quantity of materials, inspect the surface and internal quality of materials by computer vision algorithm. Secondly, artificial intelligence will be conducted to the dynamic optimization and the adjustment of construction process. If there are contradictions or conflicts in the process of construction, they will be solved by analyzing the different order of construction. Moreover, artificial intelligence can help decision-makers establish decision making model, provide various alternatives, compare and judge various schemes to achieve the maximum benefits. In addition, artificial intelligence can operate multiple elements properly such as time, investment, materials, equipment and space due to it's powerful data computing capabilities, which improves the efficiency of management significantly.

In the completion stage, firstly, we can accurately audit quantities of projects based on changes in construction with the help of BIM and artificial intelligence, knowing the way to increase profits and reduce costs. Secondly, the completion information should be added without loss and omission into BIM model for retrieval and analysis. The intelligent terminal can be used to collect data, and problems in the construction such as quality defects and risks which will be visualized in the BIM model. Thirdly, the BIM model should be maintained and updated regularly. It contains a large amount of data concerned with various specialized sections needed for operation and maintenance to ensure that the information in the model is consistent with the actual situation.

\section{Robots for 3D printing}

\subsection{The defects of robots for 3D printing}

The problem of robots for 3D printing exists in the cooperative work of numerical control between software 
and hardware. The software export control depends on the model, in many cases, it is difficult to print model accurately by $\mathrm{CNC}$ software-driven mechanical equipment. The second problem is concerned with printing process. If not based on the analysis of printed objects, the process of 3D printing will be cumbersome and inefficient, it's necessary to consider its characteristics and then optimize the process. In addition, artificial intelligence is needed to learn failed cases to deal with unexpected problems during the printing process. Taking this problem for example, it is not uncommon to see that inadequate layer adhesion results in deficiency of strength and stiffness.

\subsection{Improvement and optimization for 3D printing robots}

In order to properly plan a complex tool path, one needs to determine the dynamic mechanical response of the extruded filament material as the robot arm is moving in free-space. Numerical simulations are undertaken to illustrate the basic model and machine learning algorithm is developed to optimize the robotic system to deliver a prespecified structure [16]. A research model has been developed by Wolfs Rob, which allows 3D concrete printing to be studied in an efficient way. The model is set up in a parametric environment, combining a structural analysis with an optimization module based on the simulated annealing algorithm [17]. Gardiner employed a 6-axis gantry robot with interchangeable end effector tooling to coarsely $3 \mathrm{D}$ print and subsequently mill finish complex moulds for concrete at a construction scale [18].

To solve the problem, artificial intelligence algorithm is applied for model slicing and path planning to generate multiple schemes, then choose the best one with high efficiency and quality. After that, 3D model is imported into the numerical control system. The system deals with the model in two steps: a series of parallel 2D models are used to fit the 3D model called hierarchical slice processing, the sliced profile is converted into a printing path called path planning. After generating mechanical movement instructions, the model will be stacked layer by layer. Therefore, under the control of the planned path, robots ultimately complete the construction. In the process of printing optimization, BIM can be transformed into several processes. By using artificial intelligence algorithms and learning a large number of different process plans, the best solution will be selected after analyzing and comparing the advantages and disadvantages of various plans. This will be efficient to optimize the material consumption and reduce construction time. In addition, with the help of compute vision algorithms, robots can analyze the structure they are printing, once found wrong, they can immediately fix the problem. For instance, if there is a rough surface of the structure which can be automatically observed, robots will take measures to ensure smoothness soon.

\section{BIM platform system for 3D printing}

Integration of $\mathrm{BIM}$ method with 3D printing modeling will be effective for energy efficiency, better design, cost reduction and isolation of structure [19]. Ding explored a new BIM-based automated construction system (BIMAC) including composition of BIMAC, detail of execution setup, data issues, filling layer algorithm and shows printed example results, which are the highly customized building components [20]. Davtalab proposed a software platform for data retrieval and analysis from BIM models and utilizing it efficiently during various stages of the process [21]. Correa studied the impact of BIM in bridging the gap between design and construction and proposed a computer-aided system that translates a generic architectural project in a set of pieces to be fabricated with 3D printers [22].

System integration should be based on BIM platform, usually using the Revit API for secondary development, hardware integration should consider the matching and the convenience of regular system expansion. Network technology should be used in system integration, considering requirements of network topology, the system should adopt TCP/IP reference model.

It shows that the interaction is based on the BIM model, and the information in database is concerned with the components and objects in the model. Specifically, the four parts of main information exported into the BIM model are: design information, construction information, operation and maintenance information. Design information includes: architectural design, structural design and electrical equipment design. Construction information includes: construction process, construction quality and construction monitoring. Operation and maintenance information includes: structure and component status warning and assessment and electrical equipment status, structural testing and repair records.

The BIM model is based on cloud and can be connected with the BIM platform through the Internet and mobile Internet. All data obtained after being processed is stored in the BIM cloud, which can be inserted, extracted and modified in BIM in time to update the BIM model automatically. User interface interaction can adopt Client/Server mode or Brower/Server mode and should be used for network security measures such as encryption and hierarchical authorization. The system can grant remote access monitoring data and reports through the internet. Monitoring information management includes importing of monitoring information, exporting data in the form of graphics or files, the query of historical monitoring information and the visualization of monitoring information.

Monitoring and warning information are displayed in BIM, switching from different perspectives, transforming coordinate range, rotation, cutting and synchronization updating. The warning status information is classified into good, general and poor, by using three colors to represent the status: green, yellow and red. BIM cloud platform should be able to work with other software and have nice human-computer interaction. When one of the objects is operated in BIM, it can show the object's original data, monitoring data, changes in the curve. When loading the BIM model, it can realize parametric loading according to the user's concerns and highlight 
important details. At the same time, the conclusion and warning information of BIM monitoring system, combined with virtual data, are expressed by virtual reality technology (VR) and augmented reality technology (AR).

\section{Summary}

From above, artificial intelligence and BIM certainly provides some useful methods for the problems existing in construction 3D printing. It is foreseeable that the construction 3D printing combined with BIM and artificial intelligence will be the trend in future. Admittedly, this paper only proposes the application framework of the combination, there are still many aspects which is not discussed in depth. If they can be successfully applied to the construction $3 \mathrm{D}$ printing, it is not far to realize automatic construction.

\section{References}

1. J. Pegna, Automation in Construction, 5, 427 (1997)

2. K. Behrokh, Automation in Construction, 13, 5 (2004)

3. E. Dini, R. Nannini, M. Chiarugi, WO, EP1868793(2007)

4. R. A. Buswell, A. Thorpe, R. C. Soar, A.G.F. Gibb, Automation in Construction, 17, 923(2008)

5. C. M. Rouhana, M. S. Aoun, F. S. Faek, M. S. Eljazzar, F. R. Hamzeh, 22nd Annual Conference of the International Group for Lean Construction (IGLC, Oslo, 2014)

6. V. Mechtcherine, V. N. Nerella, BFT Magazine, 82, 150(2016)

7. F. E. Sakka, F. Hamzeh, Conference of the International Group for Lean Construction (IGLC, Heraklion, 2017).

8. J. Yang, Y. Chen, W. Huang, Y. Li, International Conference on Automation and Computing (IEEE, Huddersfield, 2017)
9. Z. Yang, J. Shen, Seventh International Symposium on Computational Intelligence and Design (IEEE, Hangzhou, 2015)

10. N. G. Makagonov, E. M. Blinova, I. I. Bezukladnikov, Young Researchers in Electrical and Electronic Engineering (IEEE, Moscow, 2017)

11. M. Wu, V. V. Phoha, Y. B. Moon, A. K. Belman, International Mechanical Engineering Congress and Exposition (ASME, Phoenix, 2016)

12. J. C. Lin, X. Wu, W. Yang, R. X. Zhao, L. G. Qiao, 2nd International Conference on New Material and Chemical Industry (IOP, Sanya, 2017)

13. M. Xia, J. Sanjayan, Materials \& Design, 110, 382(2016)

14. R. Lediga, D. Kruger, Solid State Phenomena, 263, 24(2017)

15. P. Shakor, P. Shakor, International Symposium on Automation and Robotics in Construction (IAARC, Taipei, 2017)

16. T. I. Zohdi, Computer Methods in Applied Mechanics \& Engineering, 331, 343(2018)

17. R. Wolfs, T. Salet, 22nd Workshop of the European Group of Intelligent Computing in Engineering (EGICE, Eindhoven, 2015)

18. J. B. Gardiner, S. Janssen, N. Kirchner, International Symposium on Automation and Robotics in Construction (IAARC, Auburn, 2016).

19. M. Sakin, Y. C. Kiroglu, Energy Procedia, 134, 702(2017)

20. L. Ding, R. Wei, H. Che, Procedia Engineering, 85, 123(2014)

21. O. Davtalab, A. Kazemian, B. Khoshnevis, Automation in Construction, 89, 13(2018)

22. F. Correa, F. Correa, International Symposium on Automation and Robotics in Construction (IAARC, Auburn, 2016) 